\title{
UNA CARACTERIZACIÓN SOCIODEMOGRÁFICA DE LAS UNIDADES DOMÉSTICAS EN LA CIUDAD DE MÉXICO
}

\author{
BRígida García \\ $y$ \\ OrLandina de Oliveira \\ El Colegio de México
}

\section{INTRODUCCIÓN}

LA IMPORTANCIA del hogar como unidad de estudio sociodemográfico ha sido establecida con claridad para el caso de América Latina (Burch, Lira y Lopes, 1976). ${ }^{1}$ Al considerar este tema como objeto de estudio en sí, generalmente se privilegia el análisis del tamaño, composición de parentesco, ciclo vital y otras de las características estructurales internas del hogar. Asimismo, muchas veces se juzga relevante detenerse en las diferencias que presentan dichos rasgos según algunas características que atañen al jefe del hogar (edad, sexo, ocupación, etc.).

Además de esta línea de trabajo, el énfasis en el hogar ha surgido recientemente con mayor claridad al buscar mejorar la comprensión sobre las tendencias observadas en los fenómenos sociodemográficos. Dicho interés, que no considera al hogar como objeto de estudio en sí, puede presentarse como:

a) Alternativo frente a las orientaciones que atomizan la realidad al centrarse en los individuos como unidades aisladas en vez de referirlos al grupo familiar al que pertenecen (Lira, 1976). Aquí el individuo permanecería como el principal centro de atención, pero visto desde la perspectiva de su núcleo familiar. ${ }^{2}$

1 El hogar comprende a la familia, en la medida en que este último término abarca sólo a los miembros emparentados entre sí por sangre, adopción o matrimonio. Pueden existir en el hogar personas no emparentadas entre sí, y en la medida que compartan un presupuesto común, este término se convierte en sinónimo de unidad doméstica. En caso de no existir un presupuesto común, pero sí personas no unidas por lazos de parentesco, el término hogar puede utilizarse como sinónimo de vivienda. (Lira, Introducción al estudio de la familia y el hogar, 1976.) En esta investigación (véase la nota 4) se utiliza el concepto de hogar-unidad doméstica.

2 A partir de dichas consideraciones han surgido intentos en el campo de los estudios de población por conceptualizar a la familia como mediación entre los pro- 
b) También como alternativo de las corrientes tradicionales, pero considerado el hogar como una unidad más adecuada de análisis que el individuo para el estudio de algunos fenómenos (tipologías familiares en el caso de la fecundidad, por ejemplo). Aquí lo que interesa es el comportamiento de los unos junto al de los otros en la lucha por la sobrevivencia común.

El presente trabajo se ubica dentro de la primera corriente de interés - trabajos cuyo objeto de estudio es el hogar en sí. Constituye el producto inicial de una investigación que busca mostrar la importancia del hogar como unidad de análisis para el estudio de la participación de la población en la actividad económica. Nuestra hipótesis de trabajo más general es que los vínculos económicos y sociales ${ }^{3}$ que trae aparejada la pertenencia de la mayoría de individuos a hogares determinados pueden llevar a respuestas familiares, en vez de individuales, frente a contracciones o expansiones en la demanda de fuerza de trabajo. Asimismo, se intenta demostrar que las características de la estructura interna de los hogares posibilitan u obstaculizan esa respuesta económica y dan lugar a una división determinada del trabajo entre los miembros de los mismos.

Según lo anterior, se juzga necesario efectuar una caracterización inicial de la composición de parentesco, tamaño y ciclo vital de las unidades domésticas en la ciudad de México (de manera global y según el sexo de los jefes), la cual es el objeto específico de este primer trabajo. ${ }^{4}$ Después se estudian las diferencias en estructura interna que presentan los hogares dirigidos por jefes con ubicación diferencial en la estructura económica. Posteriormente, en la parte central del trabajo se explora el condicionamiento mutuo de ambas instancias (la inserción del jefe en la estructura económica en un momento en el tiempo y las características de estructura interna de sus hogares), así como su influencia conjunta sobre la participación económica de los demás miembros del hogar.

cesos económicos y sociales globales y el comportamiento demográfico individual (Zémelman, 1977).

3 El impacto del contexto familiar no se limita a la influencia que ejerce sobre las condiciones materiales de existencia de sus miembros, al abrir diferentes alternativas o al imponer obstáculos en este sentido. La pertenencia a dicho contexto involucra además estar expuesto a un proceso de socialización, de transmisión de normas y valores.

4 Este análisis se basa en la información recolectada en la encuesta de "Migración interna, estructura ocupacional y movilidad social en el área metropolitana de la ciudad de México", patrocinada conjuntamente por El Colegio de México y el Instituto de Investigaciones Sociales de la UNAM. En este análisis se utiliza la información de la primera fase (fase $a$ ) del proyecto, que consistió en la aplicación de una cédula de carácter colectivo de tipo censal a una muestra representativa de hogares en el área metropolitana de la ciudad de México. Para mayores detalles sobre la recolección de la información y la muestra, véase Muñoz, Oliveira y Stern (1977). 


\section{CaRacterísticas SOCIODEMográficas de LAS unidades domésticas}

\section{Consideraciones generales}

Para la caracterización de las unidades domésticas de la ciudad de México se consideran algunos rasgos de la unidad propiamente dicha (composición de parentesco y tamaño) así como ciertas características del jefe que ejercen una influencia indiscutible sobre la estructura interna de los hogares: edad (indicador de ciclo vital, véase infra) y sexo.

Las características de composición y tamaño son las que con mayor frecuencia se analizan en la literatura para describir la estructura de los hogares y su evolución a través del tiempo y del espacio. Estas características adquieren importancia si se busca conocer la estructura interna de las unidades en un momento en el tiempo y no para dilucidar la problemática de la transformación de los tipos de hogar a lo largo del proceso de cambio de la sociedad, como a veces suele hacerse en la literatura (Levy, 1965; Burch, 1967). ${ }^{5}$ Como ya se mencionó se considera que la relevancia de estas características se deriva del impacto que puedan tener sobre la participación de los miembros del hogar en la actividad económica.

En lo que se refiere a las características del jefe, se utiliza la edad como una aproximación al concepto de ciclo vital, uno de los conceptos más antiguos e importantes en los campos de la sociología y de la demografía de la familia. Como señala un autor, "la idea básica es que las familias pasan a través de una secuencia de estadios característicos, tales como el matrimonio, el nacimiento de los hijos, el 'lanzamiento' de los hijos hacia sus respectivos matrimonios, el estadio 'post-hijos' y la disolución de la familia por la muerte de uno de los cónyuges. Para cada estadio el tamaño y la composición sufren transformaciones que tienen amplios efectos sociales y económicos en áreas como la vivienda, las pautas de consumo y ahorro, la actividad económica femenina y el bienestar social, que requieren ajustes sociológicos por parte de los individuos" (Kono, 1977, p. 356).

Las limitaciones de la conceptualización anterior sobre el ciclo vital de la familia (de manera principal operacionalizado por Glick, 1947;

5 El argumento sostenido en este tipo de interpretación es que se parte de familias pequeñas, y con escasas alternativas de composición más allá de la nuclear (uno o ambos padres con hijos solteros), debido a los altos niveles de mortalidad imperantes en las sociedades poco desarrolladas. Luego se formula la existencia de un periodo de transición donde puede darse la posibilidad de familias de tamaño grande, pero debido principalmente a la mayor sobrevivencia de los hijos (Burch, 1967). Esto es factible gracias al descenso de los niveles de mortalidad, producido por la incorporación de tecnología médica y sanitaria avanzada en los países en desarrollo. Por último, se alcanza el punto de partida de familias pequeñas y nucleares. en sociedades ya desarrolladas, de manera principal debido al descenso de los niveles de fecundidad. 
Glick y Parke Jr., 1965) han sido señaladas por el mismo Kono (1977). Entre ellas se pueden mencionar:

a) el esquema siempre supone una experiencia nuclear y excluye la posibilidad de familias extendidas;

b) implica que todas las familias completan la totalidad del ciclo antes de morir uno de los cónyuges. Se olvida la posibilidad de divorcio o muerte antes de ese momento;

c) Por último, conforme a este esquema sólo se enfatiza el supuesto de la familia con hijos por oposición a la pareja que no desea o no puede tenerlos, las cuales llegan a constituir un número importante en algunos países como por ejemplo Japón en 1970 (véase Kono, 1977, pp. 358359). Asimismo, deja de lado la posibilidad de hogares con jefes sin cónyuge y sin hijos (véase infra la definición de unidades "sin componente nuclear").

Debido con seguridad a las dificultades que acarrea la habilitación del esquema analizado para diferenciar las familias por ciclo vital, sobre todo en países no necesariamente caracterizados por un predominio casi absoluto de familias nucleares y con información estadística poco detallada, a menudo se emplea la edad del jefe de la familia como una aproximación al ciclo vital. Cabe aclarar que en muy pocos casos se establecen equivalencias entre los tramos de edad que se escogen y alguna conceptualización propia del ciclo bajo consideración. Glick (1947), constituye una excepción a este respecto.

A pesar de ser un indicador muy burdo, la edad del jefe ha resultado ser una aproximación útil al concepto de ciclo vital de la familia que se relaciona de manera importante con las características de tamaño y composición (Van der Tak y Gendell, 1973; Pantelides, 1976; Lira, 1976 y 1976a; Lopes, 1976; Glick, 1977). En este sentido es que se considera relevante incluir dicho rasgo en nuestra caracterización. Se considera necesario mostrar sobre todo hasta qué punto el panorama que se estructure, con base en el tamaño y composición, sufre transformaciones importantes entre unidades que se encuentran en diferentes momentos de su ciclo vital.

Acerca del sexo del jefe, existen numerosas evidencias sobre la importancia de incluirlo en una caracterización de unidades domésticas (Van der Tak y Gendell, 1973; Pantelides, 1976; Lopes, 1976; Glick, 1977). En las unidades dirigidas por mujeres, por lo general no existe cónyuge, y su composición de parentesco con frecuencia se aleja de la norma nuclear que contempla sólo la existencia de hijos solteros en el hogar.

La prevalencia de este tipo de unidades en una sociedad determinada ha llevado a diversas interpretaciones. Por ejemplo, en el caso de ciudades de países en desarrollo se afirma que su presencia constituye evidencia del "atractivo especial que tiene para las mujeres la economía citadina dominada por los servicios, y también el desventajoso estatus 
de las mujeres en una sociedad donde la unión consensual (y más el apareamiento casual) está todavía diseminado" (Van der Tak y Gendell, 1973 , p. 322). Al contrario de la formulación anterior, en países como Estados Unidos, el aumento reciente de unidades domésticas dirigidas por mujeres ha sido interpretado como un efecto de las crecientes tasas de divorcio, la decreciente ocurrencia de nuevos matrimonios y los descensos en los niveles de fecundidad que han aumentado la posibilidad de que la madre joven mantenga una vivienda separada (Glick, 1977).

En la línea de interés del presente trabajo, también existe suficiente evidencia de que los hogares dirigidos por mujeres se caracterizan por arreglos de participación económica distintos a los que prevalecen entre los hogares con jefes hombres, en especial en lo que se refiere a la participación económica femenina (Lopes, 1976).

\section{El caso de México}

Las tendencias que presentan las variables mencionadas con anterioridad para el caso del país o de la capital son casi desconocidas. Apenas se cuenta con alguna evidencia parcial en lo que toca al tamaño de los hogares. ${ }^{6}$ Según datos censales, el tamaño medio de la familia aumentó de manera continua hasta $1960: 4.1,4.5$ y 5.4 para el país y $3.4,4.2$ y 5.3 para el Distrito Federal en 1940, 1950 y 1960, respectivamente (obsérvese que la distancia que separa al Distrito Federal del país en su conjunto se acorta con el correr de los años). No obstante, a partir de 1960 la tendencia se revierte y las cifras de tamaño medio bajan al nivel de 4.9 para el país y 4.6 para el Distrito Federal.

Por desgracia, debido al procedimiento censal, en el caso de México no es posible establecer comparaciones entre las cifras citadas en el párrafo anterior y la información que usualmente se recolecta al respecto a nivel internacional (Burch 1967; Burch, Lira y Lopes, 1976). En nuestro caso, los censos definen como familia a los núcleos conyugales dentro de la vivienda. ${ }^{7}$ Estos, como es fácil deducir, tienden a tener un tamaño medio menor que los hogares o familias definidos conforme a las recomendaciones internacionales (véase la nota 1 ).

Además del problema de comparabilidad, se considera que las ten-

6 Fuera de algunas comunidades rurales estudiadas por antropólogos, la información sobre hogares en las áreas urbanas es escasa en lo que respecta a composición de parentesco, ciclo vital y características relacionadas (Nolasco, 1977; Leñero, 1968).

7 "Familia censal es el conjunto de personas que, unidas o no por parentesco, hacen vida en común bajo un mismo techo, en torno a un núcleo familiar conyugal. Por lo tanto, la familia censal comprende, además de los jefes, las esposas(os) o campañeras(os) y los hijos, a las personas con otro parentesco y a las personas sin parentesco, siempre y cuando éstas vivan en la misma vivienda que los primeros y no formen a su vez otro núcleo familiar conyugal, en cuyo caso se considerarán como otra familia en la vivienda" (véase, Censos de Población, Dirección General de Estadística, México). 
dencias descendentes que presentan los datos analizados no son confiables. Por lo menos hasta 1970, ni la fecundidad comenzaba a descender de manera apreciable, ni se contaba con alguna evidencia importante de que los niveles de mortalidad fuesen en ascenso, o que disminuyera la edad al casarse. Estos tres factores que, entre otros, pueden hacer descender el tamaño medio de la familia si cambian en el sentido señalado, mostraban más bien signos confiables de permanecer constantes (fecundidad) o de moverse en sentido inverso al señalado (mortalidad y edad al casarse). (El Colegio de México, 1970; Quilodrán, 1976).

En vista de la escasa disponibilidad de información para el caso de México, se consideran justificados los intentos que se realicen en la línea del presente análisis, para el cual se contó con información especial proveniente de una encuesta. Se analizan en primer lugar la composición y el tamaño de las unidades domésticas en la ciudad de México y sus cambios por ciclo vital en un momento en el tiempo. Más adelante se estudian las variaciones que presentan los rasgos anteriores según el sexo del jefe de los hogares.

\section{Composición de PARENTEsco y tamaño de los hogares EN LA CIUDAD DE MÉXICo}

\section{Composición de parentesco}

En el cuadro 1 se presentan las definiciones de las categorías de composición de parentesco utilizadas en nuestra descripción. Las clasificaciones de unidades domésticas nucleares, extendidas y compuestas son adaptaciones de las utilizadas en la literatura, hechas con el fin de diferenciar a los hogares según su grado de complejidad. Además, se introdujo la modalidad "sin componente nuclear". Se trata de los hogares sin componente nuclear del jefe, o sea aquellas en que el jefe no tiene pareja, ni hijos solteros. ${ }^{8}$ Estas unidades se caracterizan por un jefe que vive solo (unipersonal), o un jefe que vive con un hijo casado o con otros parientes (madre, padre, tíos, hermanos, etc.), o un jefe que vive con uno o más no parientes.

En la literatura, esta categoría de unidad doméstica es clasificada como extendida (jefe con otros parientes) o compuesta (jefe con noparientes). Las únicas "sin componente nuclear" que por lo común son diferenciadas son las unipersonales. La razón por la cual se distinguieron estos hogares como un tipo aparte es que ellos representan un

8 La categoría sin componente nuclear no implica de manera necesaria la existencia de una familia "incompleta", como suele hacerse en la literatura al tomar como modelo de las clasificaciones de composición la familia nuclear completa (Lira, 1976). En nuestro caso la ausencia de un cónyuge no es por necesidad resultado de la disolución (muerte, abandono) de una relación conyugal previamente establecida. 
Cuadro 1

Ciudad de México: Categorías de composición de parentesco Y EL GRADO DE PARTICIPACIÓN (\%) DE CADA UNO DE ELLOS

Categoría de la

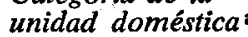
Definición $\begin{array}{ll}\text { Nuclear } & \text { Se compone de la pareja de esposos con o sin } \\ \text { hijos solteros. Incluye además al jefe solo con }\end{array}$ uno o más hijos solteros

Extendida

Está formada por una familia nuclear más algún otro pariente que no sea hijo de soltero. Este pariente puede ser un hijo casado o cualquier otro en la línea de parentesco vertical o colateral

Tipo I

Con otros parientes solos

Tipo II Con otros parientes que forman otro núcleo familiar

Compuesta

Comprende a la familia nuclear o extendida más otra $\mathbf{u}$ otras personas no emparentadas con el jefe que no sean empleadas domésticas

Sin componente nuclear del jefe

Unipersonal

Compuesta por una persona que vive sola sin parientes o no-parientes que no sean empleadas domésticas

Pluripersonal

Compuesta por un jefe sin cónyuge y sin hijos solteros que vive con otros parientes o no-parientes

Tipo I

Jefe con otros parientes o no-parientes solos.

Tipo II

Jefe con otros parientes o no-parientes que forman otro núcleo familiar

Total

Clasificación adaptada de la utilizada por Lopes (1971).

arreglo muy especial en donde posiblemente la participación económica sea distinta a la de los hogares extendidos o compuestos. En estos últimos hogares, según nuestra definición, además de haber otros parientes o no parientes, según el caso, existe la familia nuclear del jefe.

Las empleadas domésticas que viven en la casa del patrón se eliminaron de la clasificación de las unidades domésticas para evitar que su presencia modificara la composición de un hogar si se les considerara como un no pariente. Este es el caso, por ejemplo, de los hogares nucleares que son clasificados como compuestos en muchos trabajos por la presencia de la empleada doméstica. 
Como se aprecia en la distribución porcentual que figura en el cuadro 1, en la ciudad de México, como en otras ciudades latinoamericanas, existe una proporción importante, pero no mayoritaria, de unidades nucleares. Si se consideran las unidades no-nucleares en su conjunto como se hace en la literatura (véase CEPAL, 1975) puede observarse que éstas representan una proporción nada despreciable del total (38\%). Esto hace ubicar la capital del país fuera de aquel grupo de países y ciudades (en su mayoría pertenecientes a sociedades occidentales desarrolladas - véase CEPAL, 1975, pp. 176-191- aunque existen algunas excepciones importantes) donde el grupo familiar nuclear existe separado en su mayor parte de su contraparte extendido.

Sin embargo, es evidente que al encuadrar los arreglos familiares correspondientes a 1970 en la ciudad de México dentro de la dicotomía nuclear-no nuclear, se pierde mucha de la riqueza que los datos presentan en este sentido. Dichas clasificaciones excluyentes sólo muestran la importancia de las unidades nucleares, pero relegan a un lugar secundario, tal vez por ser minoritarias, una variedad de arreglos domésticos alternativos como son los propiamente extendidos (21.6\%), compuestos (3\%), y los sin componente nuclear $(13 \%)$.

La composición de parentesco varía de manera considerable al comparar unidades domésticas que están en diferentes etapas de su ciclo vital (véase el cuadro 2).$^{9}$ Las unidades nucleares alcanzan su mayor peso (alrededor de $70 \%$ ), en hogares cuyos jefes tienen entre 25 y 44 años de edad y pierden importancia entre las unidades que se encuentran en la última etapa de su ciclo vital. La participación del arreglo nuclear completo con hijos explica esta tendencia global: el nuclear sin hijos y el incompleto aumentan su participación a partir de los 45 años de edad del jefe. Lo anterior refleja el abandono del hogar por parte de los hijos o la muerte de alguno de los cónyuges, o ambas cosas.

Para las unidades "sin componente nuclear", se observa una tendencia que pudiera calificarse como contraparte de la que muestran las unidades nucleares completas con hijos y algo más parecida a la que se señaló como característica de las completas sin hijos. Por lo visto, las unidades con jefes muy jóvenes, o que cuentan con más de 45 años de edad, son campos fértiles para arreglos domésticos que se apartan de la norma nuclear que pudiera denominarse como mayoritaria. Tal vez entre los jefes jóvenes el proceso de formación de arreglos "sin componente nuclear" esté asociado a la muerte de uno de los padres; el hijo o hija soltera se hace entonces cargo de la casa. En edades más avanzadas del jefe, este tipo de arreglo posiblemente sea el resultado de la viudez o separación de la pareja en hogares en que ya no viven hijos solteros.

- Para llegar a las agrupaciones de edad que se muestran en el cuadro 2 se buscó combinar aquellos grupos más utilizados en la literatura con los que aglutinasen características de composición y tamaño más homogéneas en el caso de nuestra información. 


\section{Cuadro 2}

DisTRIBUCIÓN DE LAS UNIDADES DOMÉSTICAS SEGÚN SU COMPOSICIÓN DE PARENTESCO Y EDAD DEL JEFE

(Area Metropolitana, 1970)

\begin{tabular}{|c|c|c|c|c|c|}
\hline \multirow[b]{2}{*}{ Composición de parentemoal } & \multicolumn{5}{|c|}{ Edad del jefe } \\
\hline & $15-24$ & $25-34$ & $35-44$ & ${ }_{\text {más }}^{45}$ & Total \\
\hline Nuclear & 55.8 & 70.7 & 69.3 & 53.5 & $\underline{61: 8}$ \\
\hline Completa & 55.2 & 67.6 & 62.0 & 43.3 & $54: 7$ \\
\hline $\begin{array}{l}\text { sin hijos } \\
\text { can hijos }\end{array}$ & $\begin{array}{l}13.5 \\
41.7\end{array}$ & $\begin{array}{r}6.3 \\
61.3\end{array}$ & $\begin{array}{r}2.7 \\
59.3\end{array}$ & $\begin{array}{r}8.8 \\
34.5\end{array}$ & $\begin{array}{r}5.9 \\
47.8\end{array}$ \\
\hline Incomple ta & 0.6 & 3.1 & 7.3 & 10.2 & $7: 1$ \\
\hline Extendida & 14.1 & 18.2 & 20.7 & 25.2 & 21.6 \\
\hline $\begin{array}{l}\text { Tipo I } \\
\text { Tipo II. }\end{array}$ & $\begin{array}{r}10.4 \\
3.7\end{array}$ & $\begin{array}{r}13.8 \\
4.4\end{array}$ & $\begin{array}{r}15.6 \\
5.1\end{array}$ & $\begin{array}{l}13.2 \\
12.0\end{array}$ & $\begin{array}{r}13.8 \\
7.8\end{array}$ \\
\hline Compuesta & 1.2 & 1.9 & 2.5 & 4.1 & 3.0 \\
\hline Sin componente nuclear & 28.8 & 8.9 & 7.5 & 17.1 & 13.4 \\
\hline $\begin{array}{l}\text { Unipersonal } \\
\text { Pluripersonal }\end{array}$ & $\begin{array}{r}5.5 \\
23.3\end{array}$ & $\begin{array}{l}2.3 \\
6.6\end{array}$ & $\begin{array}{l}2.7 \\
4.8\end{array}$ & $\begin{array}{l}8.3 \\
8.8\end{array}$ & $\begin{array}{l}5.2 \\
8.2\end{array}$ \\
\hline $\begin{array}{l}\text { Tipo I } \\
\text { Tipo II }\end{array}$ & $\begin{array}{l}11.0 \\
12.3\end{array}$ & $\begin{array}{l}3.7 \\
2.9\end{array}$ & $\begin{array}{l}3.5 \\
1.3\end{array}$ & $\begin{array}{l}6.0 \\
2.8\end{array}$ & $\begin{array}{r}5.1 \\
.3 .1\end{array}$ \\
\hline Total & 99.9 & $100.0^{\circ}$ & 100.0 & 99.9 & 100.0 \\
\hline
\end{tabular}

FUENTE: Fase $A$ de la Encuesta de Migración.

a Véase en el cuadro 1 la definición de la variable composición de parentesco de la unidad doméstica.

b Los números entre paréntesis indican el número de casos.

Las unidades extendidas y compuestas registran un aumento sistemático en su participación en el total, a medida que se pasa de un ciclo vital al siguiente, aunque se observa una variante interesante entre los dos grupos de unidades extendidas. Resulta claro que la presencia de dos o más núcleos familiares dentro de este tipo de arreglo es un fenómeno más bien característico de las unidades en su última etapa del ciclo vital. Este hallazgo muestra la importancia que adquieren, para el caso de la ciudad de México, arreglos donde pudieran estar presentes tres generaciones sucesivas, las cuales constituyen la modalidad extensiva en el sentido más amplio de la palabra (véase Bock, Iutaka y Berardo, 1976).

Estos cambios en la composición de parentesco entre unidades en diferentes momentos del ciclo vital con seguridad traen aparejada una organización distinta de la participación económica de los miembros de 
la familia, y una atribución de significado diferente al papel que juega el trabajo de la mujer en diferentes contextos familiares. Dichos hallazgos muestran lo pertinente que sería la inclusión de dicho ciclo en cualquier análisis cuyo interés sea observar cómo la composición de parentesco propicia diferentes respuestas de familias frente a un mismo estímulo externo (una mayor demanda de trabajo femenino, aunado a un mayor desempleo de la mano de obra masculina, por ejemplo).

Asimismo, la existencia de una cierta diversidad de arreglos no nucleares en la ciudad de México ofrece más posibilidades de analizar cómo se da la participación económica en unidades domésticas en donde coexisten la familia nuclear del jefe con otros parientes o no parientes (extendidas y compuestas), en comparación con aquellos hogares en donde no existe el componente nuclear mencionado.

\section{Tamaño medio de las unidades domésticas}

El tamaño medio de las unidades domésticas en la muestra analizada (véase el cuadro 3) es de 5.4 miembros. Esta cifra, muy similar a la encontrada en otros países en desarrollo (Burch, 1967, Van der Tak y Gendell, 1973), diferencia claramente la ciudad de México respecto de algunos países desarrollados, en donde los tamaños medios de las unidades domésticas varían entre 3.0 y 3.3 miembros. Es posible pensar que la composición de las unidades $(24.6 \%$ de extendidas y compuestas) explique en parte este tamaño medio relativamente elevado.

Para apreciar la importancia de las unidades no nucleares en la explicación del tamaño medio de los hogares, en la literatura sobre el tema por lo general se estima la proporción que representa el tamaño medio del componente no nuclear total dentro del tamaño medio de todos los hogares (Burch, 1967). En otros casos se estudia la relación entre las varianzas de estos dos tamaños medios (Van der Tak y Gendell, 1973). Conforme al primer camino se encuentra que, para el conjunto de hogares, el peso que tiene el tamaño medio del componente no nuclear (0.9) dentro del tamaño medio total (5.4) no pasa de $16 \% .{ }^{10}$ Esta cifra, aunada a la correspondiente al tamaño medio del componente nuclear para el conjunto de unidades domésticas (4.9) refleja más bien la importancia de pautas demográficas de fecundidad elevada y relativa

10 Esta cifra fue calculada sin considerar en el tamaño medio del componente no nuclear a las unidades denominadas "sin componente nuclear". (Debido a la necesaria ambigüedad existente para definir el componente nuclear y el no nuclear de dichas unidades, fueron consideradas sólo para el cálculo del tamaño medio total). No obstante, si también se incluyen las unidades "sin componente nuclear" en el cálculo del tamaño medio del componente no nuclear -excluido el jefe, el cual constituiría el único elemento en el componente nuclear, inclusive en las unidades unipersonales - la cifra de $16 \%$ analizada en el texto sólo asciende a $18 \%$. Por el contrario, si las unidades "sin componente nuclear" se excluyen de ambos términos considerados para el porciento, éste desciende 215 . 
Cuadro 3

TAMAÑo MEDIo a DE LAS UNIDADES DOMÉSTICAS Y SUS COMPONENTES NUCLEARES $Y$ NO NUCLEARES SEGÚN SU COMPOSICIÓN DE PARENTESCO, SEXO Y EDAD DEL JEFE

(Jefes hombres, Area Metropolitana, 1970)

\begin{tabular}{|c|c|c|c|c|c|c|}
\hline \multirow{2}{*}{$\begin{array}{l}\text { Edad del jefe } \\
\text { y tamaño medio } \\
\text { de la unidad }\end{array}$} & \multicolumn{6}{|c|}{ Composición de parentesco } \\
\hline & Nuclear & Extendida & Compuesta & Subtotal & $\begin{array}{l}\text { Sin } \\
\text { componente } \\
\text { nuclear } \\
\end{array}$ & Total \\
\hline \multicolumn{7}{|l|}{$15-24$} \\
\hline $\begin{array}{l}\text { Unidad doméstica } \\
\text { componente nuclear } \\
\text { mnpontente no nuclear } \\
25-34 \\
\end{array}$ & $\begin{array}{l}3.5 \\
3.4 \\
0.1\end{array}$ & $\begin{array}{l}5.5 \\
3.3 \\
2.2\end{array}$ & $\frac{b}{b}{ }^{\prime} /$ & $\begin{array}{l}3.9 \\
3.4 \\
0.5\end{array}$ & $\begin{array}{c}3.8 \\
- \\
-\end{array}$ & $\begin{array}{l}3.9 \\
3.4 \\
0.5\end{array}$ \\
\hline $\begin{array}{l}\text { Unidad donestica } \\
\text { Componente nuclear } \\
\text { Componente no nuclear }\end{array}$ & $\begin{array}{l}4.7 \\
4.6 \\
0.1\end{array}$ & $\begin{array}{l}6.4 \\
4.5 \\
1.9\end{array}$ & $\begin{array}{l}8.3 \\
4.4 \\
3.9\end{array}$ & $\begin{array}{l}5.1 \\
4.5 \\
0.5\end{array}$ & $\frac{3.1}{-}$ & $\begin{array}{l}5.0 \\
4.6 \\
0.5\end{array}$ \\
\hline \multicolumn{7}{|l|}{$35-44$} \\
\hline $\begin{array}{l}\text { Unidad doméstica } \\
\text { Componente nuclear } \\
\text { Componente no nuclear }\end{array}$ & $\begin{array}{l}6.2 \\
6.1 \\
0.1\end{array}$ & $\begin{array}{l}7.8 \\
6.1 \\
1.7\end{array}$ & $\begin{array}{r}10.5 \\
: \quad 5 \\
4.7\end{array}$ & $\begin{array}{l}6.7 \\
6.0 \\
0.7\end{array}$ & $\begin{array}{l}2.6 \\
-\end{array}$ & $\begin{array}{l}6.4 \\
5.0 \\
0.7\end{array}$ \\
\hline $45 \mathrm{y}$ niês & & & & & \\
\hline $\begin{array}{l}\text { Unidad doméstica } \\
\text { Componente nuclean } \\
\text { Componente no nuclear }\end{array}$ & $\begin{array}{l}5.1 \\
4.9 \\
0.2\end{array}$ & $\begin{array}{l}7 \cdot 1 \\
4 \cdot 3 \\
2 \cdot 9\end{array}$ & $\begin{array}{l}8.0 \\
3.7 \\
4.3\end{array}$ & $\begin{array}{l}5.8 \\
4.8 \\
1.2\end{array}$ & $\frac{2.3}{-}$ & $\begin{array}{l}5.2 \\
4.6 \\
1.2\end{array}$ \\
\hline \multicolumn{7}{|l|}{ Total } \\
\hline $\begin{array}{l}\text { Unidad doméstiaa } \\
\text { Componente nuclear } \\
\text { Componente no nuclean }\end{array}$ & $\begin{array}{l}5.2 \\
5.1 \\
0.1\end{array}$ & $\begin{array}{l}7.1 \\
4.7 \\
2.4\end{array}$ & $\begin{array}{l}8.6 \\
4.3 \\
4.3\end{array}$ & $\begin{array}{l}5.8 \\
4.9 \\
0.9\end{array}$ & $\begin{array}{l}2.7 \\
- \\
-\end{array}$ & $\begin{array}{l}5.4 \\
4.9 \\
0.9\end{array}$ \\
\hline
\end{tabular}

baja mortalidad infantil de la sociedad mexicana en la determinación del tamaño total de las unidades.

Este sencillo procedimiento se utilizó para obtener una primera visión global con nuestros datos. No obstante, la composición de parentesco analizada sugiere que dicha aproximación puede encubrir diferencias muy importantes entre unidades domésticas de diferentes tipos que vale la pena poner de relieve.

Como puede apreciarse en las últimas tres columnas del cuadro 3, hay una interrelación clara entre el tamaño medio de la unidad doméstica y su composición de parentesco. Las unidades extendidas y las compuestas son las de mayor tamaño (7.1 y 8.6, respectivamente) y en ellas el peso del componente no nuclear representa un 25 y un $50 \%$ del tamaño total, respectivamente. No obstante, es interesante observar que, si se toma cada ciclo vital por separado, el tamaño del componente nu- 
clear no varía mucho entre unidades nucleares, extendidas y compuestas, a excepción de la etapa de 45 años y más.

Dichos resultados modifican para el caso de la ciudad de México la afirmación de Burch (1967) sobre el proceso de formación de los hogares extendidos en la mayoría de los países. Este autor afirma que ellos existen porque se da una sustitución de los hijos que se van de la casa, por otros parientes que llegan al hogar. En la ciudad de México coexisten en los hogares extendidos y compuestos parientes no nucleares con nucleares mucho antes de que el lanzamiento de los hijos tenga lugar (últimas etapas del ciclo vital).

Ahora bien, es interesante resaltar la relación curvilineal entre el tamaño medio de las unidades nucleares, extendidas y compuestas y el momento del ciclo vital analizado: las unidades alcanzan un tamaño máximo cuando el jefe tiene entre 35 y 44 años y luego disminuyen.

Esta tendencia de variación en el tamaño de las unidades domésticas de manera principal se debe al comportamiento del componente nuclear. El componente no nuclear presenta una tendencia distinta; sólo disminuye ligeramente a edades avanzadas (unidades compuestas) o inclusive aumenta, como en el caso de las unidades extendidas.

Este hallazgo refleja cómo el proceso de formación de las unidades y sus variaciones de tamaño pueden ser distintos según el tipo de hogar considerado. Los hogares que incluyen un componente nuclear reflejan en su tamaño el impacto de una serie de eventos vitales (edad al matrimonio, nacimiento de hijos y su espaciamiento, matrimonio de los hijos, etc.) que afectan a dicho componente. En los hogares que no lo incluyen ("sin componente nuclear"), el tamaño medio sigue una tendencia distinta: disminuye en forma gradual a medida que la edad del jefe es mayor, posiblemente más como resultado de la mortalidad o matrimonio de miembros solteros que del número de hijos o espaciamiento entre ellos, como ocurre en los otros tipos de unidades. Estos resultados sugieren que para entendẹr las variaciones del tamaño medio de las unidades domésticas es necesario considerar a la vez la composición de parentesco y el ciclo vital de las mismas.

\section{DiFERENCIAS DE COMPOSICIÓN Y TAMAÑo DE LAS UNIDADES DOMÉSTICAS SEGÚN EL SEXO DEL JEFE}

En lo que se refiere a la composición de parentesco de las unidades, las tendencias de los hogares dirigidos por hombres son en esencia las mismas que se encontraron para la población total (véase el cuadro 2). Esto se debe al hecho de que dichas unidades domésticas constituyen una parte muy significativa del total $(84 \%)$. Sin embargo, se presenta un hallazgo interesante que debe ser debidamente ponderado: la importante prevalencia de arreglos nucleares incompletos y "sin componente 


\section{Cuadro 4}

Distribución DE LAS UNIDADES DOMÉSTICAS SEGÚN SU COMPOSICIÓN DE PARENTESCO, SEXO Y EDAD DEL JEFE

(Jefes hombres, Área Metropolitana, 1970)

\begin{tabular}{|c|c|c|c|c|c|}
\hline \multirow[b]{2}{*}{ Composiciôn de parentescoal } & \multicolumn{5}{|c|}{ Edad dei jefe } \\
\hline & $15-24$ & $25-34$ & $35-44$ & $\begin{array}{r}45 \mathrm{y}^{\mathrm{y}} \\
\mathrm{ras}\end{array}$ & TotaI \\
\hline Nuclear & $\underline{58.1}$ & $\underline{71.3}$ & 71.3 & 62.2 & 66.9 \\
\hline Completa & 58.1 & 71.3 & 70.6 & 59.4 & 65.6 \\
\hline $\begin{array}{l}\text { sin hijos } \\
\text { con hijos }\end{array}$ & $\begin{array}{l}14.2 \\
43.9\end{array}$ & $\begin{array}{r}6.6 \\
64.7\end{array}$ & $\begin{array}{r}3.1 \\
67.5\end{array}$ & $\begin{array}{l}11.9 \\
47.5\end{array}$ & $\begin{array}{r}8.2 \\
57.4\end{array}$ \\
\hline Incompleta & - & - & $0: 7$ & 2.8 & 1.3 \\
\hline Extendida & $\underline{13.5}$ & $\underline{19.1}$ & 20.0 & 26.9 & $\underline{21.8}$ \\
\hline $\begin{array}{l}\text { Tipo I } \\
\text { Tipo II }\end{array}$ & $\begin{array}{r}10.3 \\
3.2\end{array}$ & $\begin{array}{r}14.5 \\
4.6\end{array}$ & $\begin{array}{r}15.1 \\
4.9\end{array}$ & $\begin{array}{l}14.2 \\
12.7\end{array}$ & $\begin{array}{r}14.2 \\
7.6\end{array}$ \\
\hline Compuesta & 0.7 & $\underline{2.0}$ & 2.9 & 4.0 & 2.9 \\
\hline Sin componente ruciear & $\underline{27.9}$ & 7.7 & 5,9 & 6.9 & 8.5 \\
\hline $\begin{array}{l}\text { Unipersonal } \\
\text { Iluripersonal }\end{array}$ & $\begin{array}{r}5.2 \\
22.7\end{array}$ & $\begin{array}{l}1.7 \\
6.0\end{array}$ & $\begin{array}{l}2.0 \\
3.9\end{array}$ & $\begin{array}{l}3.5 \\
3.3\end{array}$ & $\begin{array}{l}2.8 \\
5.7\end{array}$ \\
\hline $\begin{array}{l}\text { Tipo I } \\
\text { Tipo II }\end{array}$ & $\begin{array}{r}9.8 \\
12.9\end{array}$ & $\begin{array}{l}3.1 \\
2.9\end{array}$ & $\begin{array}{l}2.6 \\
1.3\end{array}$ & $\begin{array}{l}2.1 \\
1.2\end{array}$ & $\begin{array}{l}3.1 \\
2.6\end{array}$ \\
\hline TotaI D & 100.2 & 100.1 & 100.1 & 100.0 & 100.1 \\
\hline & $(155)$ & $(546)$ & (550) & (749) & $(2000)$ \\
\hline
\end{tabular}

Fuente: Fase $A$ de la Encuesta de Migración.

a Véase a la definición de esta variable en el cuadro 1.

b Los números entre paréntesis indican el número de casos.

nuclear" en la última etapa del ciclo vital, se ve disminuida de manera notable en el cuadro 4 referente a hombres.

Esta tendencia sugiere analizar con mayor cuidado la información de las unidades con jefes mujeres que presenta algunas peculiaridades interesantes (véase el cuadro 5). Alrededor de tres cuartas partes de estas unidades son nucleares incompletas y "sin componente nuclear", y un $21 \%$ ha sido ubicado como extendido. Este hallazgo deja claro la importancia de arreglos domésticos alternativos al patrón nuclear tradicional (padres e hijos solteros) entre las jefes mujeres. (Dicho resultado ha sido encontrado en otras ciudades latinoamericanas. Van der Tak y Gendell, 1973; Pantelides, 1976; Lira, 1976a).

Con respecto a las diferencias por ciclo vital, es interesante observar que las unidades nucleares incompletas disminuyen de manera considerable en la etapa representada por 45 años y más. Por el contrario, los arreglos sin componente nuclear, y los extendidos del segundo tipo, 


\section{Cuadro 5}

DistribuCIÓN DE LAS UNIDADES DOMÉSTICAS SEGÚN SU COMPOSICIÓN DE PARENTESCO, SEXO Y EDAD DEL JEFE

(Jefes mujeres, Area Metropolitana, 1970)

\begin{tabular}{|c|c|c|c|c|c|c|}
\hline \multirow{2}{*}{\multicolumn{3}{|c|}{ Composicion de parentesco a ! }} & \multicolumn{4}{|c|}{ Edad del jefe } \\
\hline & & & \multirow{2}{*}{$\frac{15-34}{54.3}$} & \multirow{2}{*}{$\begin{array}{c}35-44 \\
55.2\end{array}$} & \multirow{2}{*}{$\begin{array}{c}45 \mathrm{y} m a \mathrm{~s} \\
30.5\end{array}$} & \multirow{2}{*}{ Total } \\
\hline Nuclear & & & & & & \\
\hline Completa & & & & & & \\
\hline $\begin{array}{l}\text { sin hijos } \\
\text { con hijos }\end{array}$ & & & - & $\overline{1.3}$ & 0.7 & $\begin{array}{l}0.5 \\
0.3\end{array}$ \\
\hline Incompleta & & & 54.3 & 53.9 & 29.8 & 35.7 \\
\hline Extendida & & & 5.8 & $\underline{25.6}$ & 20.9 & 20.6 \\
\hline $\begin{array}{l}\text { Tipo I } \\
\text { Tipo II }\end{array}$ & & & $\begin{array}{l}2.9 \\
2.9\end{array}$ & $\begin{array}{r}19.2 \\
6.4\end{array}$ & $\begin{array}{l}10.6 \\
10.3\end{array}$ & $\begin{array}{r}11.7 \\
8.9\end{array}$ \\
\hline Compuesta & & & 2.9 & - & 4.3 & 3.3 \\
\hline Sin componente & nuclear & & 35.2 & 19.3 & 44.3 & 38.7 \\
\hline $\begin{array}{l}\text { Unipersonal } \\
\text { Pluripersonal }\end{array}$ & & & $\begin{array}{l}14.3 \\
20.9\end{array}$ & $\begin{array}{r}7.7 \\
11.6\end{array}$ & $\begin{array}{l}20.9 \\
23.4\end{array}$ & $\begin{array}{l}17.7 \\
21.0\end{array}$ \\
\hline $\begin{array}{l}\text { Tipo I } \\
\text { Tipo II }\end{array}$ & & & $\begin{array}{r}20.0 \\
2.9\end{array}$ & $\begin{array}{r}10.3 \\
1.3\end{array}$ & $\begin{array}{r}16.3 \\
7.1\end{array}$ & $\begin{array}{r}15.4 \\
5.6\end{array}$ \\
\hline Total & $\therefore$ & & $\begin{array}{c}100.2 \% \\
(35)\end{array}$ & $\begin{array}{r}100.1 \\
(78)\end{array}$ & $\begin{array}{l}100.0 \\
(282)\end{array}$ & $\begin{array}{l}100.2 \\
(395)\end{array}$ \\
\hline
\end{tabular}

Fuente: Fase $A$ de la Encuesta de Migración.

a Véase la definición de esta variable en el cuadro 1.

b Los números entre paréntesis indican el número de casos.

aumentan en ese grupo de edad. Estos hechos pueden evidenciar, de manera indirecta, el importante abandono de los hijos de los hogares dirigidos por mujeres, o también, en el caso de los extendidos, su unión y creación de núcleos conyugales adicionales bajo el mismo techo. Asimismo, el notable aumento de unidades "sin componente nuclear" en la etapa mencionada del ciclo vital (45 años y más) puede ser resultado de la menor mortalidad femenina en comparación con la masculina que tiene lugar en el país en edades avanzadas.

En lo que se refiere a las diferencias de tamaño medio entre unidades domésticas con jefes hombres y aquellas con jefes mujeres, se observa (véanse los cuadros 6 y 7) que las tendencias encontradas para la población total (véase el cuadro 3) también se manifiestan de igual forma en la población masculina. Son las unidades domésticas con jefes mujeres las que presentan diferencias importantes: dichas unidades tienen un tamaño medio menor, de manera principal como resultado del tamaño 
pequeño de su componente nuclear (Pantelides, 1976). Esta tendencia posiblemente esté asociada al predominio de familias nucleares incompletas y "sin componente nuclear" entre jefes mujeres.

Por otra parte, la tendencia (encontrada en la población total) a disminuir que registra el tamaño del componente nuclear en las unidades nucleares y extendidas con jefes de 45 años en adelante, es más marcada en las unidades con jefes mujeres. Esto refuerza las hipótesis formuladas en torno a los datos de composición sobre el mayor abandono de los hijos, o creación de nuevas unidades familiares, en aquellos hogares dirigidos por mujeres.

\section{Cuadro 6}

TAMAÑO MEDIO ${ }^{a}$ DE LAS UNIDADES DOMÉSTICAS Y SUS COMPONENTES NUCLEARES Y NO NUCLEARES SEGÚN SU COMPOSICIÓN DE PARENTESCO, SEXO Y EDAD DEL JEFE

(Jefes hombres, Area Metropolitana, 1970)

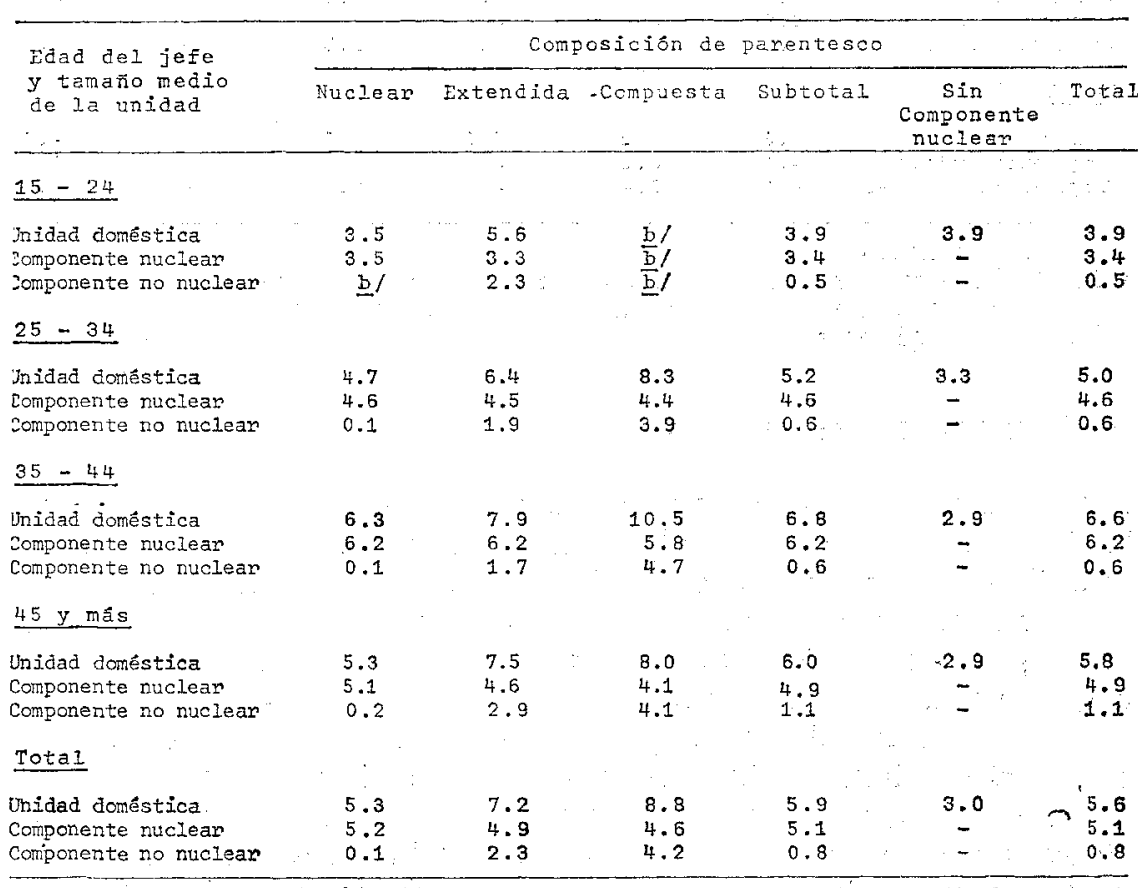

FUENTE: La misma del cuadro 2.

a Los tamaños medios de la unidad y de su componente no nuclear incluyen a las empleadas domésticas que residen en la casa de sus patrones.

b Menos de cinco casos. 


\section{Cuadro 7}

TAMAÑo MEDIO ${ }^{a}$ DE LAS UNIDADES DOMÉSTICAS Y SUS COMPONENTES NUCLEARES $Y$ NO NUCLEARES SEGÚN SU COMPOSICIÓN DE PARENTESCO, SEXO Y EDAD DEL JEFE

(Jefes mujeres, Ârea Metropolitana, 1970)

\begin{tabular}{|c|c|c|c|c|c|c|}
\hline \multirow{2}{*}{$\begin{array}{l}\text { Edad del jefe } \\
\text { y tamano medio de } \\
\text { la unidad }\end{array}$} & \multicolumn{6}{|c|}{ Composición de parentesco } \\
\hline & Nuclean & Extendida & Compuesta & SubtotaI & $\begin{array}{l}\text { Sin } \\
\text { componente } \\
\text { nuclean }\end{array}$ & Total \\
\hline $15-34$ & & - & & & & \\
\hline $\begin{array}{l}\text { Unidad doméstica } \\
\text { Componente nucleas } \\
\text { Componente no nuclean } \\
35-44\end{array}$ & $\begin{array}{l}4.4 \\
4.3 \\
0.1\end{array}$ & $\frac{\mathrm{b} /}{\mathrm{b} /}$ & $\frac{b}{b} /$ & $\begin{array}{l}4.4 \\
4.3 \\
0.1\end{array}$ & $\stackrel{2.3}{-}$ & $\begin{array}{l}3.6 \\
4.3 \\
0.1\end{array}$ \\
\hline $\begin{array}{l}\text { Unidad donêstica } \\
\text { Componente nucleas } \\
\text { Componente no nuclean }\end{array}$ & $\begin{array}{l}5.2 \\
5.1 \\
0.1\end{array}$ & $\begin{array}{l}7 \cdot 3 \\
5.5 \\
1.3\end{array}$ & $\overline{-}$ & $\begin{array}{l}5.8 \\
5.2 \\
0.6\end{array}$ & 1.9 & $\begin{array}{l}5.1 \\
5.2 \\
0.6\end{array}$ \\
\hline $45 \mathrm{ymas}$ & & & & & & \\
\hline $\begin{array}{l}\text { Unidad doméstica } \\
\text { Componente nuclear } \\
\text { Componente no nuclear }\end{array}$ & $\begin{array}{r}3.8 \\
3.6 \\
: 0.2\end{array}$ & $\begin{array}{l}5.9 \\
3.2 \\
2.7\end{array}$ & $\begin{array}{l}8.0 \\
3.2 \\
4.8\end{array}$ & $\begin{array}{l}4.9 \\
3.4 \\
1.5\end{array}$ & $\begin{array}{c}2.4 \\
- \\
-\end{array}$ & $\begin{array}{l}3.8 \\
3.4 \\
1.5\end{array}$ \\
\hline Total & & & & & & \\
\hline $\begin{array}{l}\text { Unidad domestica } \\
\text { Componente nuclear } \\
\text { Componente no nuclear }\end{array}$ & $\begin{array}{l}4.3 \\
4.1 \\
0.1\end{array}$ & $\begin{array}{l}5.2 \\
3.8 \\
2.4\end{array}$ & $\begin{array}{l}7.8 \\
3.1 \\
4.7\end{array}$ & $\begin{array}{l}5.2 \\
3.9 \\
1.3\end{array}$ & $\begin{array}{l}2.3 \\
-\end{array}$ & $\begin{array}{l}4.0 \\
3.9 \\
1.3\end{array}$ \\
\hline
\end{tabular}

FUENTE: La misma del cuadro 2.

a Los tamaños medios de la unidad y de su componente no nuclear incluyen a las empleadas domésticas que residen en la casa de sus patrones.

b Menos de cinco casos.

\section{Consideraciones finales}

Los hallazgos sobre composición y tamaño permiten concluir que para la ciudad de México en 1970 la familia nuclear y de gran tamaño medio es el tipo de hogar más común. No obstante, la proporción de arreglos familiares no nucleares no es despreciable, de manera principal en el subconjunto de hogares dirigidos por mujeres. El tamaño medio de estos hogares no nucleares (extendidos y compuestos) es mayor que el de las unidades nucleares debido a que a un componente nuclear de tamaño similar al de ellas, se añade la presencia de otros parientes.

Frente a hallazgos semejantes, algunos autores han buscado explicaciones, tanto a nivel de las características de la estructura interna de la unidad, como de las características socioeconómicas del jefe. ${ }^{11}$ Así, por QII.:

11 Asimismo, con frecuencia se relacionan los cambios en los procesos sociales con los familiares. "La hipótesis fundamental (que se debate) sería que existe una 
ejemplo, Van der Tak y Gendell (1973) encontraron para el caso de Guatemala que la perpetuación de la familia extendida de manera principal se debía a la gran proporción de familias encabezadas por varones o mujeres sin cónyuge; a partir de este hallazgo, es sólo de manera marginal que se considera la influencia de otras variables en el ámbito socioeconómico.

Por el contrario, Bock, Iutaka y Berardo (1976) dedican todo su análisis a determinar el peso de las variables de edad, posición social, origen extranjero, migración y movilidad social en la explicación de la existencia de grupos nucleares y extendidos en Buenos Aires, Río de Janeiro y Santiago. ${ }^{12}$ Pero no se hace consideración explícita del aspecto de estructura interna de la familia - su carácter "completo e incompleto" - tan enfatizado en el caso de Guatemala.

Puede observarse que la búsqueda de explicaciones en ambos sentidos es limitada al no contar con una visión histórica de los cambios ocurridos en la estructura familiar de las sociedades en cuestión. Debido a que se afrontó este mismo tipo de limitación, no se abunda mayormente en la búsqueda de "factores" que estarían dando cuenta de la coexistencia de arreglos nucleares y no nucleares en la ciudad de México en un momento determinado. Mas bien orientan futuros análisis hacia la búsqueda de los tipos de arreglos familiares que coexisten al interior de diferentes grupos sociales y para configurar asi los "contextos" que podrían condicionar la participación económica de los miembros del hogar, $y$ en particular el trabajo femenino.

\section{REFERENCIAS}

Bock, Wilbur, Iutaka Sugiyama y Felix M. Berardo (1976), "La familia nuclear y extendida en áreas urbanas de la Argentina, el Brasil y Chile", en La familia como unidad de estudio demográfico, Santiago de Chile, CELADE.

Burch, Thomas (1967), "The Size and Structure of Families. A Comparative Analysis of Census Data", American Sociological Review, 32(3): 347-63, junio. Burch, Thomas y Murray, Gendell (1976), "Estructura de la familia extendida y

relación inversa entre la industrialización y urbanización y la familia extendida. De este modo, a medida que se desarrolla una sociedad, la familia extendida tiende a ser reemplazada por una nuclear independiente. Del mismo modo, dentro de una misma sociedad las familias extendidas prevalecerán más en las áreas rurales que en las urbanas, en las regiones menos desarrolladas que en las más avanzadas" (Lira, 1976, p. 263). Diversos autores han cuestionado a través de trabajos empíricos la validez de esta hipótesis y ha recibido mayor apoyo una proposición de Levy (1965) que sin duda sólo atañe al aspecto más superficial del fenómeno bajo estudio: "los diseños generales y la naturaleza de las estructuras actuales de la familia han sido virtualmente idénticos en ciertos aspectos estratégicos en todas las sociedades conocidas en la historia del mundo, por lo menos para el $50 \%$ de los miembros de esas sociedades" (pp. 41-42).

12 Estos autores no encontraron una configuración común en la asociación de los factores considerados para las tres ciudades. 
fecundidad: algunos aspectos conceptuales y metodológicos", en La familia como unidad de estudio demográfico, Santiago de Chile, CELADE.

CELADE (1976), La familia como unidad de estudio demográfico, Santiago de Chile.

CEPAL (1975), Conferencia Mundial de Población, México, Fondo de Cultura Económica.

El Colegio de México (1970), Dinámica de la Población de México, México, cEeD. García Brígida y Orlandina de Oliveira (1978), "Reflexiones teóricas-metodológicas sobre el estudio de las relaciones entre el trabajo de la mujer y la fecundidad en la ciudad de México", Investigación Demográfica en México, MéXico, CONACYT.

Glick, Paul (1947), "The Family Cycle", American Sociological Review, 12: 164174.

Glick, Paul y Robert Parke Jr. (1965), "New Approaches in Studying the Life Cycle of the Family", Demography, 2 (1), 187-202.

Glick, Paul (1977), "Dimensions of the Fields of Family Demography", International Population Conference, International Unión for the Scientific Study of Population, México.

Kono, Shigemi (1977), "The Concept of the Family Life Cycle as a Bridge Between Demography and Sociology", International Population Conference, International Union for the Scientific Study of Population, México.

Leñero, Luis (1976), Investigación de la familia en México, México, Ed. IMES.

Levy, Marion (1965), Aspects of Analysis of Family Structure, New Jersey, Princeton University Press.

Lira, Luis Felipe (1976), "Aspectos demográficos de la familia en una provincia de Chile según el Censo de 1970", en La familia como unidad de estudio demográfico, Santiago de Chile, CELADE.

(1976a), "Características socioeconómicas y estructurales de las familias en la ciudad de Santiago de Chile, 1970", La familia como unidad de estudio demográfico, Santiago de Chile, CELADE.

Lopes, V. F. (1971), El uso del computador para la obtención de datos sobre familia, a base de la información del Censo de Población, Santiago de Chile, CELADE.

- (1976), La familia en el Brasil según el Censo de Población de 1960", La familia como unidad de estudio demográfico, Santiago de Chile, CELADE.

Muñoz, Humberto, Orlandina de Oliveira y Claudio Stern (1977), Migración y desigualdad social en la ciudad de México, México, IsUnam y colmex.

Nolasco, Margarita (1977), "Proceso de urbanización y estructura familiar", ponencia presentada en el 1er. Simposio Mexicano Centroamericano de Investigación sobre la Mujer, México.

Pantelides, Edith (1976), "El hogar como unidad de análisis de los datos censales: importancia y posibilidad", La familia como unidad de estudio demográfico, Santiago de Chile, CELADE.

Quilodrán, Julieta (1976), "Análisis de la nupcialidad en las áreas rurales de México", México (Mimeo.).

Van der Tak, Jean y Murray Gendell (1973), "The Size and Structure of Residential Families, Guatemala City, 1964", Population Studies, 27 (1): 305-322, julio.

Zemelman, Hugo (1977), Problemas en la explicación del comportamiento reproductivo", México (Mimeo.) 